\title{
AVALIAÇÃO FÍSICO-QUÍMICA E SENSORIAL DE CACHAÇA ENVELHECIDA EM BARRIS DE EUCALIPTO
}

\author{
E. K. D. VIÉGAS ${ }^{1}$, S. H. da CRUZ ${ }^{1}$, U. de A. LIMA $^{1}$ e A. R. ALCARDE ${ }^{1}$ \\ ${ }^{1}$ Universidade de São Paulo, Departamento de Agroindústria, Alimentos e Nutrição \\ E-mail para contato: ellenviegas@usp.br
}

\begin{abstract}
RESUMO - A madeira desempenha um papel importante na qualidade da cachaça. Grande parte dos barris utilizados no envelhecimento da cachaça são de carvalho europeu (Quercus sp), de difícil aquisição. O armazenamento da cachaça em barris de madeira causa estabilização química e confere características de envelhecimento, provocando alterações nas propriedades sensoriais. $\mathrm{O}$ objetivo deste trabalho foi avaliar o uso de doze espécies de eucalipto para a confecção de barris destinados ao envelhecimento de cachaça. A bebida envelhecida por 12 meses foi avaliada sensorialmente utilizando o teste triangular e teste de aceitação. Foram realizadas determinações de cor, acidez volátil, graduação alcoólica. Os resultados obtidos demonstraram boa aceitação para todas as amostras, no entanto não houve diferença significativa em relação aos atributos da cachaça envelhecida nos barris de eucalipto. Todas as amostras apresentaram aumento acentuado da coloração. Houve maior redução de acidez nas amostras de cachaça envelhecidas em barris de Eucalyptus microcorys. A madeira de eucalipto pode ser considerada um material adequado para confecção de barris utilizados no envelhecimento de cachaça.
\end{abstract}

\section{INTRODUÇÃO}

A cachaça vem conquistando espaço em diversas classes sociais, sendo responsável por gerar renda e emprego. A cachaça é definida com sendo uma denominação típica e exclusiva para aguardente de cana produzida no Brasil, com graduação alcoólica de 38 a $48 \%$ em volume, a $20^{\circ} \mathrm{C}$, com características sensoriais peculiares (BRASIL, 2005).

De um montante de 1,5 bilhões de litros produzidos por ano, o mercado externo absorve em torno de $1 \%$ (IBRAC, 2014). Apesar do crescimento dos últimos anos, a participação das exportações de aguardente no total da produção nacional ainda é pequena. A maior parte da aguardente destina-se ao mercado interno. Convicta que a bebida detém significativo potencial de mercado, a Câmara Setorial da Cachaça estima que haverá uma elevação de 1 para $10 \%$ de volume produzido para exportação da aguardente até 2018 (Ministério da Agricultura, Pecuária e Abastecimento - MAPA, 2013).

A qualidade sensorial de uma bebida é nitidamente afetada durante o armazenamento ou envelhecimento, em razão direta da permanência do produto em barris de madeira. As características de cor, aroma e sabor da bebida envelhecida evoluem de forma que estão relacionadas com o tipo de madeira e tratamentos prévios, à geometria e história do barril e ao tempo de armazenamento (SILVA JÚNIOR, 1999). 


\section{9 a 22 de outubro de 2014 \\ Florianópolis/SC}

Nonato et al. (2001) demonstraram que os ésteres e alcoóis superiores produzidos durante o processo fermentativo contribuem para o aroma de bebidas fermentadas; o aroma de frutas presente no vinho, brandy, saquê e em outras bebidas é atribuído a compostos como o caproato de etila (maçã), acetato de isoamila (banana) e acetato de 2-feniletil (frutas, flores e mel).

A qualidade das bebidas alcoólicas é julgada por sua cor, aroma e sabor. Tais características sensoriais dependem da matéria-prima utilizada, das condições de fermentação e destilação e do processo de maturação e envelhecimento. Uma bebida pode ser engarrafada e comercializada logo após a destilação; no entanto por apresentar sabor ardente e seco (DIAS, 1997) deverá ser armazenada ou envelhecida.

O armazenamento da bebida em barris de madeiras após certo tempo influi na composição química, aroma, sabor e cor do destilado. O período de maturação ou envelhecimento é de fundamental importância para eliminar ou minimizar a presença de substâncias que proporcionam aroma e sabor desagradáveis (PIGGOTT, 1989; LIMA, 1992).

Embora o carvalho seja a madeira tradicional e mais usada para fabricar recipientes para armazenamento de bebidas destiladas, a utilização de madeiras de eucaliptos para produção de barris revela-se particularmente interessante. Dentre as diversas espécies do gênero Eucalyptus, existe uma grande variação de cor, odor, sabor, permeabilidade, densidade, resistência mecânica, durabilidade natural e manipulação. Existe a possibilidade, dentro desta amplitude de características, de algumas delas se apresentarem adequadas para a fabricação de barris destinados ao armazenamento de aguardentes, além do fato de serem espécies de reflorestamento, de rápido crescimento e alta produtividade (MORI et al., 2003).

Tendo em vista que o Brasil é o maior produtor de eucalipto fora de seu habitat natural e, dado que são cultivadas diferentes espécies, esta pesquisa visa avaliar o uso de madeira de eucalipto para a confecção de barris destinados ao envelhecimento de cachaça; desenvolvendo uma bebida com características que se possa considerar totalmente nacional.

\section{MATERIAL E MÉTODOS}

Os experimentos foram conduzidos no Setor de Açúcar e Álcool do Departamento de Agroindústria, Alimentos e Nutrição da Escola Superior de Agricultura "Luiz de Queiroz" ESALQ/USP. Os barris de 20 litros de capacidade foram confeccionados na Tanoaria Casa Bernardo, localizada na cidade de Piracicaba - SP, utilizando sete espécies de eucaliptos (Tabela 1) provenientes do Laboratório de Propriedades Físicas e Processamento da Madeira do Departamento de Ciências Florestais da ESALQ/USP.

Tabela 1 - Espécies de eucaliptos utilizadas para confecção dos barris

\begin{tabular}{lll}
\hline E. citriodora & E. pellita & E. resinifera \\
E. maculata & E. phaeotricha & E. saligna \\
E. microcorys & E. pilularis & E. tereticornis \\
E. paniculata & E. pyrocarpa & E. torelliana
\end{tabular}




\section{9 a 22 de outubro de 2014 \\ Florianópolis/SC}

A aguardente com 46, $3^{\circ} \mathrm{GL}$, gentilmente cedida pela Empresa Capuava, Piracicaba - SP, foi armazenada nos diferentes barris após saturação com água. Como tratamento testemunha, uma amostra da aguardente foi armazenada em recipiente de vidro nas mesmas condições do experimento. Os barris permaneceram na adega do Setor de Açúcar e Álcool da ESALQ/USP à temperatura média de $26^{\circ} \mathrm{C}$ com pouca luminosidade por doze meses. Periodicamente, amostras da cachaça foram coletadas para as análises físico-químicas.

\subsection{Análises físico-químicas da cachaça}

Amostras de $100 \mathrm{~mL}$ de aguardente foram retiradas, de cada barril e transferidas para frascos de fechamento hermético, para a realização de análises químicas. A estocagem das amostras em frascos de fechamento hermético tem a finalidade de mantê-las, supostamente, inalteradas até as análises.

Teor alcoólico: analisado por densitometria em densímetro digital (ANTON PAAR 4500).

Coloração: mensurada em espectrofotômetro (FEMTO 700S) a $420 \mathrm{~nm}$ para um percurso óptico de $1 \pm 0,001 \mathrm{~mm}(\mathrm{CHATONET}, 1995)$.

Acidez volátil: $10 \mathrm{~mL}$ da amostra foram colocados no borbulhador e $250 \mathrm{~mL}$ de água destilada no gerador de vapor do aparelho de destilação. Recolheu-se $100 \mathrm{~mL}$ do destilado, que foram titulados com hidróxido de sódio $0,1 \mathrm{~N}$, em presença de fenolftaleína $1 \%$. A acidez volátil foi expressa em miligramas de ácido acético por $100 \mathrm{~mL}$ de amostra $(\mathrm{mg} / 100 \mathrm{~mL})$.

\subsection{Análise sensorial}

Amostras da aguardente armazenada nos diferentes barris de eucalipto e a amostra testemunha, após 12 meses de armazenamento, foram analisadas sensorialmente por teste triangular de diferença e por teste afetivo de aceitação das amostras em relação aos atributos aroma, sabor e aparência. Os testes foram realizados em sala com temperatura ambiente, com 70 provadores não treinados de ambos os sexos, maiores de 21 anos, recrutados no Campus "Luiz de Queiroz". Para cada provador foram oferecidos $20 \mathrm{~mL}$ de cada amostra codificada.

Os testes foram realizados após o consentimento por escrito de todos os participantes e os procedimentos foram aprovados pelo Comitê de Ética em Pesquisa com seres humanos da ESALQ/USP.

Teste triangular de diferença: amostras de aguardente provenientes de 12 espécies de madeira foram avaliadas. Cerca de $20 \mathrm{~mL}$ de três amostras codificadas (sendo duas iguais e uma diferente) foram apresentadas simultaneamente em copos transparentes de vidro. O provador foi instruído a indicar qual das amostras era a diferente. (DUTCOSKY, 1996; CARDELLO, 2000).

Teste afetivo de aceitação: as amostras foram apresentadas, de forma seqüencial em copos transparentes de vidro, codificados com algarismos de três dígitos; e, analisadas em relação aos atributos aroma, sabor e aparência, mediante escala hedônica de nove pontos (STONE e SIDEL, 1993). Na ficha utilizada também foi avaliada a atitude de compra das amostras apresentadas.

\section{RESULTADOS E DISCUSSÃO}




\subsection{Caracterização das amostras armazenadas em barris de eucalipto}

Aguardente com teor alcoólico de 46,3 (\%, v/v) foi armazenada em barris de diferentes espécies de eucalipto. As alterações no teor alcoólico, cor e acidez volátil estão apresentadas na Tabela 2, Figuras 1 e 2.

Teor alcoólico: Durante o período de armazenamento, ocorreu uma diminuição na graduação alcoólica das amostras mantidas nos barris de eucalipto, exceto para a amostra armazenada em barril de E. citriodora que apresentou comportamento estatisticamente igual a amostra testemunha, armazenada em recipientes de vidro e, cujo teor alcoólico permaneceu inalterado. A cachaça armazenada em barril de E. microcorys apresentou uma redução de $4 \%$ no teor alcoólico, enquanto para a amostra armazenada em barril E. saligna a perda foi de 13\%; a aguardente armazenada em barril de E. pilularis foi a que apresentou maior diminuição no teor alcoólico durante um ano de armazenamento (Tabela 2).

Tabela 2 - Teor alcoólico (\%, v/v) das amostras de aguardente, durante armazenamento em barris confeccionados com diferentes madeiras de eucalipto.

\begin{tabular}{lcccc}
\hline \multirow{2}{*}{ Amostras } & \multicolumn{4}{c}{ Tempo de armazenamento } \\
\cline { 2 - 5 } Testemunha & $\mathbf{0}$ & 3 meses & 6 meses & 12 meses \\
\hline E. citriodora & $46,3 \mathrm{aA}$ & $46,3 \mathrm{aA}$ & $46,3 \mathrm{aA}$ & $46,3 \mathrm{aA}$ \\
\hline E. maculata & $46,3 \mathrm{aB}$ & $47,97 \mathrm{aA}$ & $45,55 \mathrm{aBC}$ & $45,25 \mathrm{aC}$ \\
\hline E. microcorys & $46,3 \mathrm{aA}$ & $46,99 \mathrm{abA}$ & $41,76 \mathrm{eC}$ & $44,45 \mathrm{abB}$ \\
\hline E. paniculata & $46,3 \mathrm{aA}$ & $46,50 \mathrm{bcA}$ & $41,96 \mathrm{eB}$ & $41,88 \mathrm{deB}$ \\
\hline E. pellita & $46,3 \mathrm{aA}$ & $45,11 \mathrm{deB}$ & $43,34 \mathrm{dC}$ & $42,84 \mathrm{cdC}$ \\
\hline E. phaeotricha & $46,3 \mathrm{aA}$ & $46,97 \mathrm{abA}$ & $44,45 \mathrm{bcB}$ & $43,67 \mathrm{bcB}$ \\
\hline E. pilularis & $46,3 \mathrm{aA}$ & $43,56 \mathrm{fB}$ & $41,96 \mathrm{eC}$ & $34,89 \mathrm{hD}$ \\
\hline E. pyrocarpa & $46,3 \mathrm{aA}$ & $44,31 \mathrm{efB}$ & $43,5 \mathrm{cdC}$ & $41,44 \mathrm{efD}$ \\
\hline E. resinifera & $46,3 \mathrm{aA}$ & $45,25 \mathrm{deB}$ & $44,82 \mathrm{abBC}$ & $44,12 \mathrm{bC}$ \\
\hline E. saligna & $46,3 \mathrm{aA}$ & $45,53 \mathrm{cdA}$ & $42,24 \mathrm{eB}$ & $39,99 \mathrm{gC}$ \\
\hline E. tereticornis & $46,3 \mathrm{aA}$ & $44,80 \mathrm{deB}$ & $43,35 \mathrm{dC}$ & $43,02 \mathrm{cC}$ \\
\hline E. torelliana & $46,3 \mathrm{aA}$ & $44,72 \mathrm{deB}$ & $42,69 \mathrm{deC}$ & $40,60 \mathrm{fgD}$ \\
\hline
\end{tabular}

* Médias seguidas pela mesma letra minúscula não diferem estatisticamente entre si na coluna e médias seguidas pela mesma maiúscula não diferem estatisticamente entre si na linha.

O comportamento em relação à graduação alcoólica foi, provavelmente, devido à passagem de vapores de água e etanol, por infiltração e evaporação, através dos poros da madeira. Elevada umidade relativa $(70 \%)$ em determinadas épocas do ano favoreceu uma maior evaporação de etanol (BORUFF, RITTSCHOF, 1959; SILVA JÚNIOR, 1999). As perdas parciais de álcool e água ao 
longo do período de armazenamento é resultado da difusão na atmosfera e depende da temperatura e espessura das aduelas (ONISHI et al., 1977; YOSHIZAWA et al., 1981).

Cor: A amostra testemunha não apresentou alteração de cor após os 12 meses de armazenamento, diferentemente das amostras armazenadas nos diferentes barris (Figura 1). A aguardente armazenada em barril de E. phaeotricha foi a que apresentou maior coloração, seguida pelos barris de E. resinífera e E. pyrocarpa.

$\mathrm{O}$ aumento observado em todas as amostras se deve a maior extração de compostos da madeira. Segundo Singleton (1995), os taninos, um dos menores constituintes da madeira, são responsáveis pelo progressivo escurecimento da cor do destilado armazenado. Outros fatores também interferem na coloração adquirida pela aguardente armazenada em barris de madeira, tais como a classificação botânica e origem da madeira que compõe o barril, os tratamentos sofridos antes do primeiro uso, o tamanho ou volume do barril, a existência de defeitos na madeira, da absorção de componentes adversos à qualidade sensorial desejada ou mesmo da presença de contaminantes de usos anteriores (MIRANDA et al., 2006).

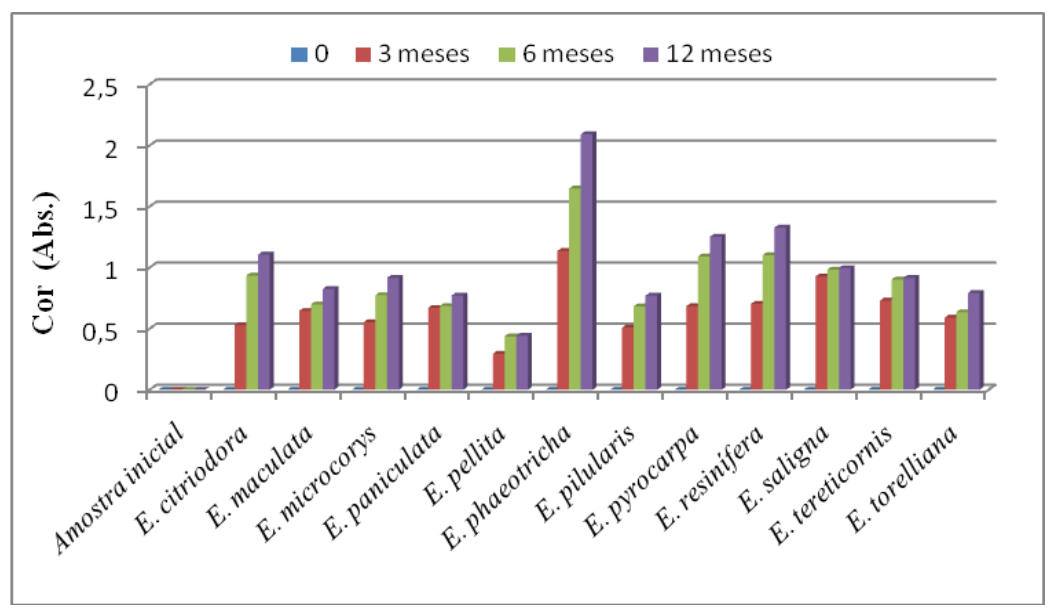

Figura 1 - Cor das amostras de aguardentes armazenadas em barris de diferentes espécies de eucalipto.

Acidez volátil: A acidez da aguardente tem influência direta no que se refere às propriedades organolépticas de uma bebida. A legislação em vigor estipula $150 \mathrm{mg}$ de ácido acético por 100mL de álcool anidro como valor máximo para a acidez volátil.

Os valores de acidez volátil durante armazenamento da aguardente em barris de diferentes espécies de eucalipto mostrou que todas as amostras apresentaram aumento da acidez volátil após um ano de armazenamento exceto para àquela armazenada em barril de E. microcorys, que reduziu para 10,14 mg de ácido acético/100mL de aguardente (Figura 2). A amostra testemunha permaneceu em 10,6 mg de ácido acético/100mL de aguardente. 
Tal redução pode ser devido à transformação do ácido acético em acetaldeído, favorecido pelas reações de oxidação como consequência de maior penetração do oxigênio do meio externo para o interior do barril, ou então a uma maior evaporação do ácido acético (SILVA JÚNIOR, 1999).

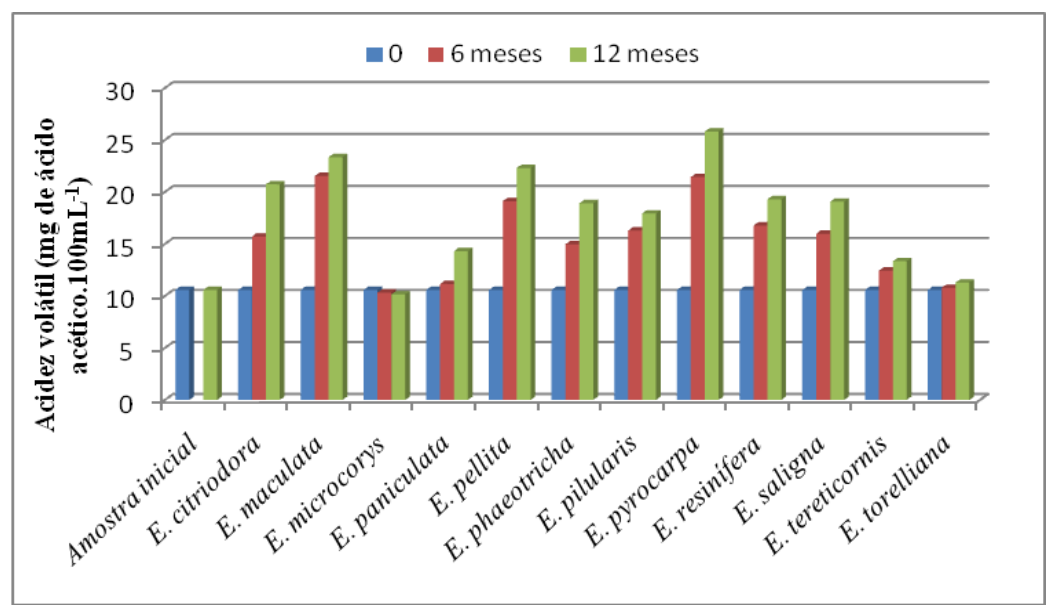

Figura 2 - Acidez volátil (mg de ácido acético. $100 \mathrm{~mL}^{-1}$ de aguardente) para as amostras armazenadas em barris de eucalipto.

Os barris foram confeccionados com madeiras novas e, portanto podem extrair maior quantidade de substâncias solúveis, características de cada madeira na aguardente, e assim proporcionar maior alteração das propriedades originais do produto do que em recipientes já estabilizados, fato que pode justificar as modificações na acidez volátil.

\subsection{Análise sensorial}

Teste de aceitação: Os valores das médias dadas pelos provadores, em função do tempo de armazenamento, com relação aos atributos aroma, sabor, cor e impressão global estão apresentados na Tabela 3. As amostras não apresentaram diferença significativa de aceitação com relação aos atributos cor, sabor, aroma e impressão global.

Tabela 3 - Médias de aceitação das amostras armazenadas por um ano.

\begin{tabular}{l|c|c|c|c}
\hline \multicolumn{1}{c|}{ Amostras } & Aroma & Sabor & Imp. global & Cor \\
\hline E. citriodora & $6,9 \mathrm{a}$ & $6,6 \mathrm{a}$ & $6,9 \mathrm{a}$ & $7,2 \mathrm{a}$ \\
\hline E. maculata & $8 \mathrm{a}$ & $7,3 \mathrm{a}$ & $7,5 \mathrm{a}$ & $7,9 \mathrm{a}$ \\
\hline E. microcorys & $6,81 \mathrm{a}$ & $6,6 \mathrm{a}$ & $6,9 \mathrm{a}$ & $7,5 \mathrm{a}$ \\
\hline E. paniculata & $6,4 \mathrm{a}$ & $6,3 \mathrm{a}$ & $6,6 \mathrm{a}$ & $7 \mathrm{a}$ \\
\hline E. pellita & $7,41 \mathrm{a}$ & $6,4 \mathrm{a}$ & $6,5 \mathrm{a}$ & $7,9 \mathrm{a}$ \\
\hline E. phaeotricha & $7,7 \mathrm{a}$ & $7,2 \mathrm{a}$ & $7,7 \mathrm{a}$ & $8 \mathrm{a}$ \\
\hline
\end{tabular}




\section{9 a 22 de outubro de 2014 \\ Florianópolis/SC}

\begin{tabular}{l|c|c|c|c}
\hline E. pilularis & $6,9 \mathrm{a}$ & $5,9 \mathrm{a}$ & $6,8 \mathrm{a}$ & $7,8 \mathrm{a}$ \\
\hline E. pyrocarpa & $7 \mathrm{a}$ & $5,4 \mathrm{a}$ & $6,7 \mathrm{a}$ & $8,3 \mathrm{a}$ \\
\hline E. resinifera & $6,8 \mathrm{a}$ & $6,1 \mathrm{a}$ & $6,6 \mathrm{a}$ & $7,8 \mathrm{a}$ \\
\hline E. saligna & $7 \mathrm{a}$ & $6,7 \mathrm{a}$ & $6,8 \mathrm{a}$ & $7,8 \mathrm{a}$ \\
\hline E. tereticornis & $6,5 \mathrm{a}$ & $7,1 \mathrm{a}$ & $7 \mathrm{a}$ & $8 \mathrm{a}$ \\
\hline E. torelliana & $6,9 \mathrm{a}$ & $6,7 \mathrm{a}$ & $7,1 \mathrm{a}$ & $7,7 \mathrm{a}$ \\
\hline
\end{tabular}

Os testes afetivos são utilizados quando se necessita conhecer o quanto o consumidor gosta ou desgosta um determinado produto, baseado em uma escala hedônica. Dos valores relativos de aceitabilidade se pode inferir a preferência (MEILGAARD et al., 1991).

Estudos já demonstraram que o envelhecimento da aguardente de cana em barris de madeira promove o aumento da aceitação e mudanças favoráveis no perfil sensorial. Com o decorrer do tempo de envelhecimento ou armazenamento, novas características sensoriais são desenvolvidas, como aroma e sabor de madeira, aumento da intensidade e duração da doçura, coloração amarela e a diminuição significativa da agressividade e do aroma e sabor alcoólico (CARDELLO e FARIA, 1999).

De acordo com as justificativas apresentadas pelos provadores nas fichas de avaliação, a melhor aceitação da aguardente armazenada em barris de eucalipto está baseada em seu aroma e sabor mais suave em relação à amostra testemunha. Os dados do teste afetivo associado aos comentários dos provadores nos levou a concluir a inviabilidade de prosseguir com o armazenamento das amostras por mais tempo, haja visto que os barris são de 20L, havendo maior área de contato entre a madeira e a bebida. Caso esse tempo de contato seja prolongado, a bebida pode se tornar intragável, com acentuação de amargor, depreciando o produto.

Apesar de não haver diferença estatística entre as amostras, 77\% dos provadores expressaram intenção de compra para as amostras armazenadas nos barris de E. maculata e E. phaeotricha; vale ressaltar que os testes foram conduzidos com provadores não treinados.

Teste triangular: Neste teste, 70 provadores participaram das análises, obtendo-se 36 respostas corretas. Ao consultar a tabela de significância para teste triangular, observamos que o número mínimo de respostas para estabelecer uma diferença significativa é de 31, podemos então afirmar que existe diferença sensorial significativa entre as amostras. Porém, os provadores não especificaram a diferença entre as amostras.

\section{CONCLUSÃO}

Doze amostras de eucalipto, E. citriodora, E. maculata, E. microcorys, E. paniculata, E. pellita, E. phaeotricha, E. pilularis, E. pyrocarpa, E. resinifera, E. saligna, E. tereticornis e E. torelliana, mostraram-se aptas para confecção de barris para armazenamento de aguardente. Os resultados obtidos demonstraram boa aceitação para todas as amostras, no entanto não houve diferença significativa em relação aos atributos da cachaça envelhecida nos barris de eucalipto. Todas as amostras apresentaram aumento acentuado da coloração. Houve maior redução de acidez nas 


\section{9 a 22 de outubro de 2014 \\ Florianópolis/SC}

amostras de cachaça envelhecidas em barris de E. microcorys. A madeira de eucalipto pode ser considerada um material adequado para confecção de barris a serem utilizados no envelhecimento de cachaça.

\section{REFERÊNCIAS}

BORUFF, C. S.; RITTSCHOF, L. A. Effects of barreling proof on the aging of American whiskeys. J. Agri. Food Chem. v. 7, p. $630-633,1959$.

BRASIL. Ministério da Agricultura, Pecuária e Abastecimento. Instrução Normativa no 13, de 29 de junho de 2005. Regulamento técnico para fixação dos padrões de identidade e qualidade para aguardente de cana e para cachaça. Diário Oficial da República Federativa do Brasil, Brasília - DF, 30 jun. 2005. p. 3.

CARDELlO, H. M. A. B.; FARIA, J. B. Análise tempo-intensidade de características sensoriais de aguardente de cana durante o envelhecimento em tonel de carvalho. Rev. Ciênc. e Tecnol. de Alim., São Paulo, n. 33, 1999. CARDELlO, H. M. A. B.; FARIA, J. B. Análise de aceitação de aguardentes da cana por testes afetivos e mapas de preferência interno. Bol. da Soc. Bras. de Ciênc e Tecnol. de Alim., Campinas v. 20, p. 32 - 36, 2000. CHATONET, P. Tese (Doutorado) - l’Universite de Bourdeaux II, Bourdeaux, 1995.

DIAS, S. M. B. C. Belo Horizonte. Dissertação (Mestrado) - Faculdade de Farmácia, Universidade Federal de Minas Gerais (UFMG). 1997.

DUTCOSKY, S. D. Análise sensorial de alimentos. Curitiba: Champagnat, 1996. 126p.

INSTITUTO BRASILEIRO DA CACHAÇA - IBRAC. Cachaça na mídia. Disponível em: www.ibraccachacas.org. Acesso em março/2014.

LIMA, U. A. Produção nacional de aguardentes e potencialidades dos mercados internos e externos. In: MUTTON, M. J. R.; MUTTON, M. A. (Ed.). Aguardente de cana: produção e qualidade. Jaboticabal: FUNEP, p.54-98. 1992.

MEILGAARD, M.; CIVILlE, G. V.; CARR, B. T. Sensory evalution techniques. 3 ed. New York: CRC Press, 387p. 1999.

MINISTÉRIO DA AGRICULTURA, PECUÁRIA E ABASTECIMENTO - MAPA. Projeções do agronegócio. Disponível em: www.agricultura.gov.br. Acesso em: março/2014.

MIRANDA, M. B.; MARTINS, N. G. S.; BEllUCO, A. E. S.; HORII, J.; ALCARDE, A. R. Qualidade química de cachaças e de aguardentes brasileiras. Ciênc. e Tecnol. de Alim., Campinas, v. 27, nº 4, p. 897 901, 2006.

MORI, F. A.; MENDES, L. M.; TRUGILHO, P. F.; CARDOSO, M. G. Utilização de eucaliptos e de madeiras nativas no armazenamento de aguardente de cana-de-açúcar. Ciênc. e Tecnol. de Alim., v. 23, p. 396-400, 2003. NONATO, E. A.; CARAZZA F.; SILVA F.C.; CARVALHO C.R.; de L. CARDEAL, Z. A headspace solidphase microextraction method for the determination of some secondary compounds of Brazilian sugar cane spirits by gas chromatography. Jou. Agric. Food Chem., v. 49, p. 3533-3539, 2001.

ONISHI, M.; GUYMON, J. F.; CROWELL, E. A. Changes in some volatile constituents of brandy during aging. Am. J. Enol. Vitic. v. 28, p. $152-158,1977$.

PIGGOTT, J. R. C.; SHARP, R. C.; DUNCAN, R. E. B. The Science and Technology of Whiskies. New York: Longman Scientific \& Technical, 1989. 410 p.

SILVA JÚNIOR, L. S. Dissertação (Mestrado em Ciência dos alimentos). Universidade Estadual Paulista "Júlio de Mesquita Filho" - Faculdade de Ciências Farmacêuticas. Araraquara - SP, 1999.

SINGLETON, V. L. Maturation of wines and spirits: comparations, facts and hypotheses. Am. J. Enol. Vitic., v. 46, p. $98-115,1995$.

STONE, H.; SIDEL, J. L. Sensory evaluation practices. 2nd ed. San Diego: Academic Press, 1993. 338p.

YOSHIZAWA, K.; MOMOSE, H.; HASUO, T. Substances evaporated through barrel during aging of whisky. Nippon Nogeikagaku Kaishi, v. 55, p. 1063 - 1068, 1981. 